\title{
Vassiliev invariants of quasipositive knots
}

\author{
Sebastian Baader
}

\begin{abstract}
Quasipositive knots are transverse intersections of complex plane curves with the standard sphere $S^{3} \subset \mathbb{C}^{2}$. It is known that any Alexander polynomial of a knot can be realized by a quasipositive knot. As a consequence, the Alexander polynomial cannot detect quasipositivity. In this paper we prove a similar result about Vassiliev invariants: for any oriented knot $K$ and any natural number $n$ there exists a quasipositive $\operatorname{knot} Q$ whose Vassiliev invariants of order less than or equal to $n$ coincide with those of $K$.
\end{abstract}

\section{Introduction}

A quasipositive braid is a product of conjugates of a positive standard generator of the braid group. If a link can be realized as the closure of a quasipositive braid, then we call it quasipositive. When Rudolph introduced quasipositive links (in [Rud83]), he showed that they could be realized as transverse $\mathbb{C}$-links, i.e. as transverse intersections of complex plane curves with the standard sphere $S^{3} \subset \mathbb{C}^{2}$. Here a complex plane curve is any set $f^{-1}(0) \subset \mathbb{C}^{2}$, where $f(z, w) \in \mathbb{C}[z, w]$ is a non-constant polynomial. Conversely, every transverse $\mathbb{C}$-link is a quasipositive link, as was recently proved by Boileau and Orevkov [BO01]. For a thorough introduction into this subject, we refer the reader to Rudolph's text book on the knot theory of complex plane curves [Rud04]. Quasipositive knots have some extreme properties that allow us to determine their four-dimensional genera (see [Rud93]). In contrast, we shall prove that quasipositivity cannot be detected by Vassiliev invariants of bounded degree. For this purpose, we use yet another description of quasipositive knots, which is based upon Seifert diagrams.

Theorem 1. For any oriented knot $K$ and any natural number $n$ there exists a quasipositive knot $Q$ whose Vassiliev invariants of order less than or equal to $n$ coincide with those of $K$.

This is related to a result of Rudolph [Rud82], who showed that any Alexander polynomial can be realized by a quasipositive knot. We also mention that Theorem 1 was formulated as a question by Stoimenow in [Sto03]. The following corollary is an immediate consequence of Theorem 1.

Corollary. It is impossible to decide whether a given knot in the standard sphere in $\mathbb{C}^{2}$ is isotopic to a transverse intersection of a complex plane curve with this sphere by using Vassiliev invariants of bounded degree.

\section{2. $C_{n}$-moves and quasipositivity}

The proof of Theorem 1 is based on a construction of Ohyama, who showed that any finite number of Vassiliev invariants can be realized by an unknotting number one knot (see [Ohy00]). His construction involves certain $C_{n}$-moves, which were defined by Habiro in [Hab94] (see also [Hab00]

Received 25 July 2005, accepted in final form 21 November 2005.

2000 Mathematics Subject Classification 57M25.

Keywords: Vassiliev invariants, quasipositive knots, complex plane curves.

This journal is (c) Foundation Compositio Mathematica 2006. 


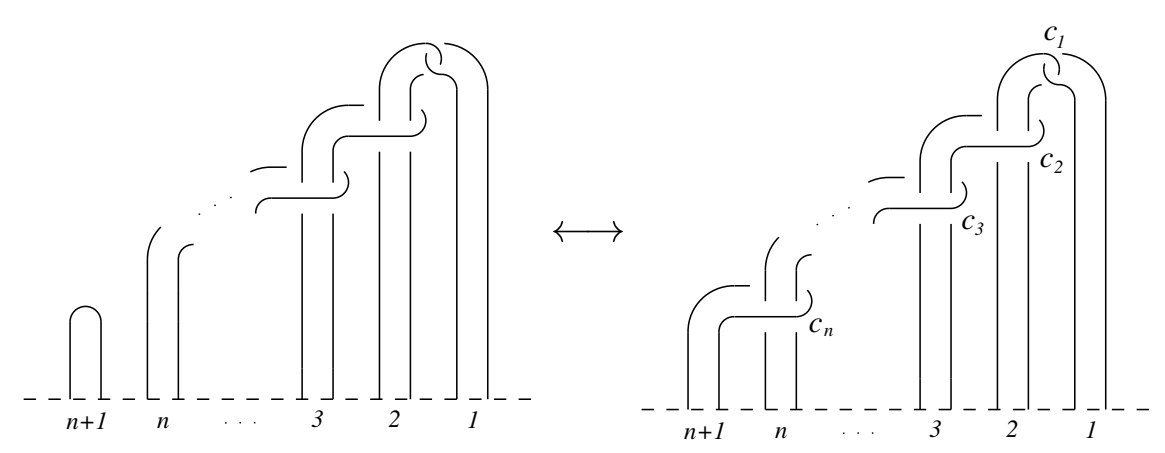

FiguRE 1. $C_{n}$-move.
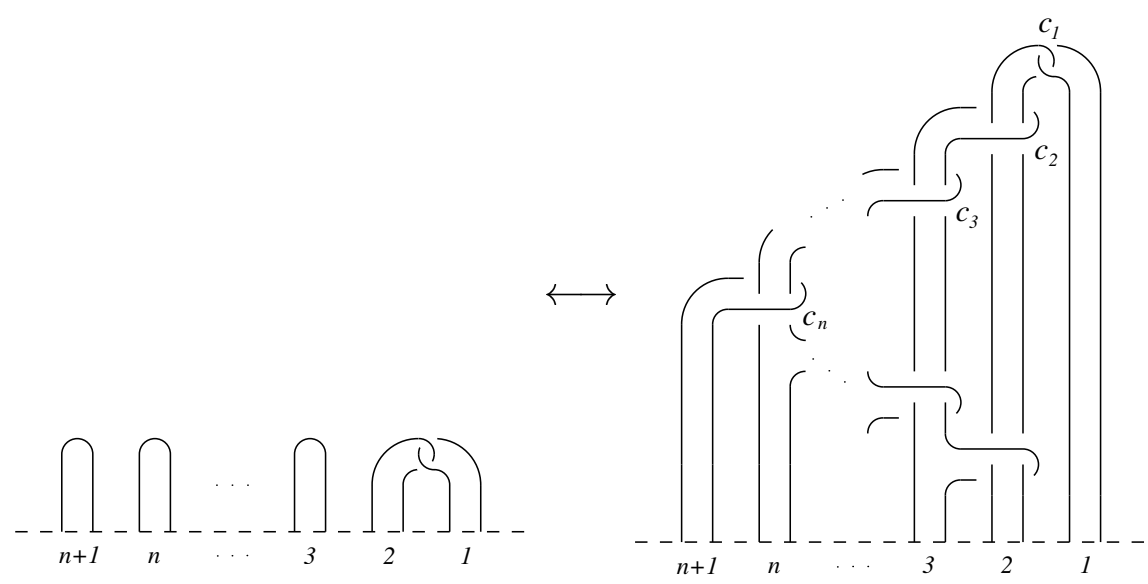

Figure 2. Special $C_{n}$-move.

and [OTY02]). A $C_{n}$-move is defined diagrammatically in Figure 1. It takes place in a section with $2(n+1)$ endpoints or $(n+1)$ strands. The strands are numbered from 1 to $n+1$ and are all connected outside the indicated section, since they belong to one knot $K$. Going along $K$ according to its orientation, starting at the first strand, we encounter the other strands in a certain order, which depends on how the strands are connected outside the indicated section. This order defines a permutation, say $\sigma \in S_{n}$, of the numbers $2,3, \ldots, n+1$.

In [OT99], Ohyama and Tsukamoto explain the effect of a $C_{n}$-move on Vassiliev invariants of order $n$. Their result [OT99, Theorem 1.2] implies the following.

(1) A $C_{n}$-move does not change the values of Vassiliev invariants of order less than $n$.

(2) Let $K$ and $\widetilde{K}$ be two knots that differ by one $C_{n}$-move, and $v_{n}$ any Vassiliev invariant of order $n$. Then $v_{n}(K)-v_{n}(\widetilde{K})$ depends only on the permutation $\sigma \in S_{n}$ defined by the cyclic order of the $(n+1)$ strands of the section where the $C_{n}$-move takes place, and on the product of the signs of the crossings $c_{1}, c_{2}, c_{3}, \ldots, c_{n}$ (see Figure 1 ).

The last statement of (2) follows from the proof of Theorem 1.2 in [OT99].

Remark. In the proof of Theorem 1, we will use special $C_{n}$-moves, as shown in Figure 2. Nevertheless, we will not need the fact that every $C_{n}$-move can be expressed as a sequence of special $C_{n}$-moves.

The proof of Theorem 1 is also based on a diagrammatical description of quasipositivity, which was introduced in [Baa05]. Since the definition in [Baa05] is not quite accurate, we shall explain it in detail here. 


\section{VASSILIEV INVARIANTS OF QUASIPOSITIVE KNOTS}

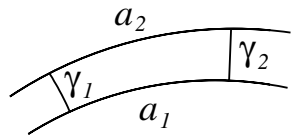

Figure 3. Rectangle.

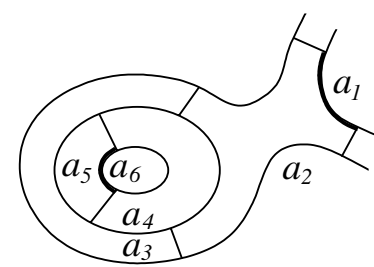

FiguRE 4. Sequence of rectangles.

A Seifert diagram is a special presentation for a knot. Starting from a planar knot diagram, we smooth all crossings in an oriented way and replace all positive (negative) crossings by a + (-) sign. Thus, a Seifert diagram is a collection of embedded circles with signs in the plane. A rather complicated Seifert diagram is shown below in Figure 6, together with its corresponding knot diagram.

Let $D$ be a Seifert diagram in the plane. Further, let $a_{1}, a_{2}$ be two arcs on adjacent Seifert circles of $D$, i.e. two closed connected subsets of adjacent Seifert circles. We say that $a_{1}$ and $a_{2}$ define a rectangle in $D$, if there exist two disjoint paths $\gamma_{1}, \gamma_{2}$ in the complement of $D$ (i.e. $\stackrel{\circ}{\gamma}_{1}, \stackrel{\circ}{2}_{2}$ are disjoint from all Seifert circles, from all crossings and from one another) that connect the endpoints of $a_{1}$ and $a_{2}$ in a way that gives rise to a rectangle, as shown in Figure 3. We denote this rectangle by $R\left(a_{1}, a_{2}\right)$.

Now let $\alpha, \beta$ be two arcs on arbitrary Seifert circles of $D$. We say that $\beta$ is inside $\alpha$, if either $\beta \subset \alpha$ or there exists a finite sequence of rectangles $R\left(a_{1}, a_{2}\right), R\left(a_{3}, a_{4}\right), \ldots, R\left(a_{2 n-1}, a_{2 n}\right)$ with $a_{1}=\alpha, a_{2 n}=\beta$ and $a_{2 k+1} \subset a_{2 k}, 1 \leqslant k \leqslant n-1$. For example, in Figure $4, a_{6}$ is inside $a_{1}$.

Definition. A Seifert diagram $D$ is quasipositive, if its set of crossings can be partitioned into single crossings and pairs of crossings, such that the following three conditions are satisfied:

(1) each single crossing is positive;

(2) each pair of crossings consists of one positive and one negative crossing joining the same two Seifert circles;

(3) if $\left(c_{1}, \bar{c}_{1}\right)$ and $\left(c_{2}, \bar{c}_{2}\right)$ are two pairs of crossings, then there exist two arcs $\alpha_{1}$ and $\alpha_{2}$ on Seifert circles of $D$, such that $\alpha_{i}$ connects the two crossings $c_{i}, \bar{c}_{i}(i=1,2)$ and either $\alpha_{1}$ is inside $\alpha_{2}$ or $\alpha_{2}$ is inside $\alpha_{1}$.

Pairs of crossings in a quasipositive Seifert diagram are called conjugating pairs of crossings.

Examples.

(i) Positive knots have quasipositive Seifert diagrams.

(ii) A Seifert diagram obtained from a braid diagram is quasipositive, if and only if it is quasipositive in Rudolph's sense. Indeed, if a Seifert diagram has concentric Seifert circles and our conditions (1)-(3) are satisfied, then we can immediately see the conjugating words in the braid group. 


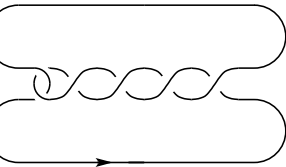

Figure 5. The knot $5_{2}$.

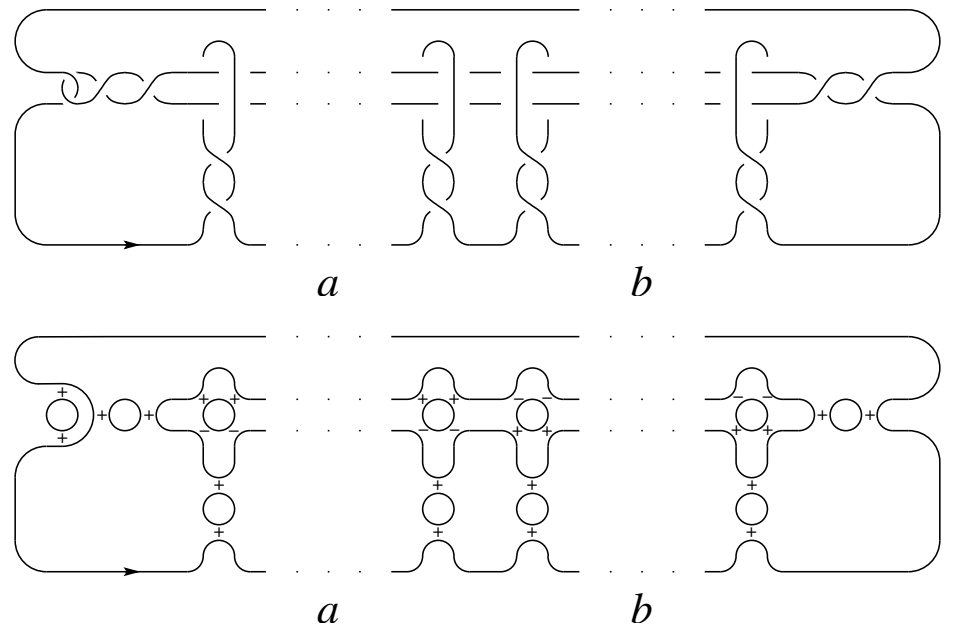

Figure 6. Diagram of $Q_{1}$.

Remark. In [Baa05], we require a weaker condition than (3). However, then it is not so clear whether quasipositive braid diagrams are quasipositive in Rudolph's sense, although this is claimed there. However, we can copy the proof of the lemma on p. 265 in [Baa05] (verbatim!) for the above notion of quasipositivity, in order to get the following equivalence with Rudolph's notion of quasipositivity.

Proposition. A knot is quasipositive in Rudolph's sense, if and only if it has a quasipositive Seifert diagram.

\section{Proof of Theorem 1}

Starting from the diagram of the positive twist knot $5_{2}$ shown in Figure 5 , we construct a quasipositive knot $Q$ with the desired properties by applying several $C_{i}$-moves, $2 \leqslant i \leqslant n$, step by step.

In the first step, we construct a quasipositive knot $Q_{1}$ whose Vassiliev invariants of order two (the Casson invariant) equals that of $K$. Choose natural numbers $a$ and $b$, such that $v_{2}(K)=2+a-b$. Here $v_{2}(K)$ is the Casson invariant of $K$. Using these two numbers, we define a knot $Q_{1}$ diagrammatically, as shown in Figure 6.

By construction, we have

$$
v_{2}\left(Q_{1}\right)=2+a-b=v_{2}(K) .
$$

This follows easily by one application of the following relation for the Casson invariant of knots:

$$
v_{2}(Y)-v_{2}(\searrow)=l k()()
$$

Indeed, a crossing change at the clasp on the left-hand side of the diagram of $Q_{1}$ produces a trivial knot, and the linking number $l k$ of the corresponding link equals $2+a-b$. Moreover, $Q_{1}$ is a quasipositive knot, since its Seifert diagram at the bottom of Figure 6 is quasipositive. 

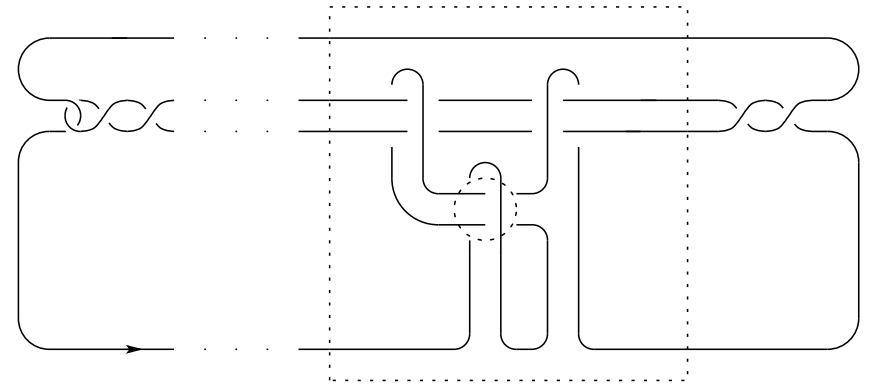

FiguRE 7. Standard pattern for a $C_{3}$-move.
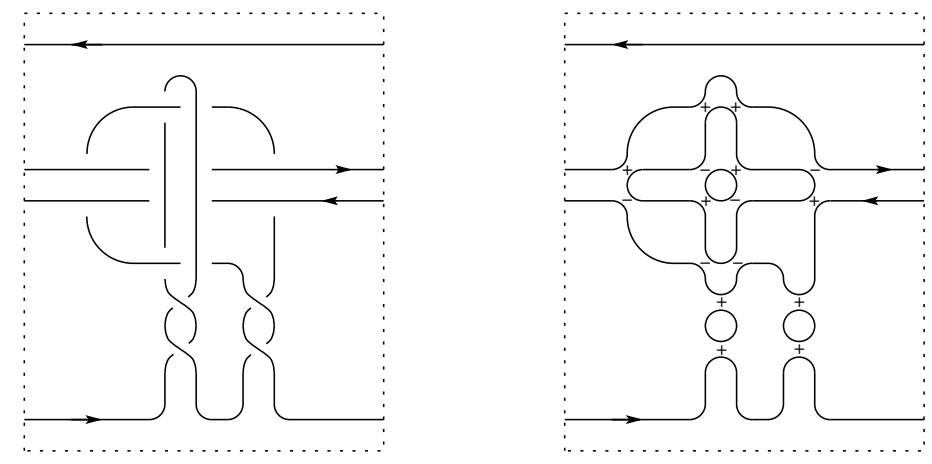

Figure 8. Quasipositive pattern for a $C_{3}$-move.

In the second step, we arrange the Vassiliev invariants of order three. Since 'all' the Vassiliev invariants of order less than or equal to two of $Q_{1}$ and $K$ coincide (i.e. $v_{2}\left(Q_{1}\right)=v_{2}(K)$ ), we conclude that $Q_{1}$ and $K$ are related by a sequence of $C_{3}$-moves. This is Habiro's result for $n=2$ (see [Hab00]). Let $K_{1}=Q_{1}, K_{2}, \ldots, K_{l}=K$ be a sequence of knots, such that two succeeding knots are related by a $C_{3}$-move. Our aim is to replace this sequence of knots by a sequence of quasipositive knots $\widetilde{K}_{1}=Q_{1}, \widetilde{K}_{2}, \ldots, \widetilde{K}_{l}$, such that

$$
v_{3}\left(\widetilde{K}_{i+1}\right)-v_{3}\left(\widetilde{K}_{i}\right)=v_{3}\left(K_{i+1}\right)-v_{3}\left(K_{i}\right),
$$

$1 \leqslant i \leqslant l-1$. By Ohyama and Tsukamoto's result, $\left|v_{3}\left(K_{2}\right)-v_{3}\left(Q_{1}\right)\right|$ depends only on the permutation $\sigma \in S_{3}$ defined by the cyclic order of the four strands of the section where the $C_{3}$-move takes place, as explained above. From this viewpoint, i.e. if we are only interested in the change of the Vassiliev invariants of order three, there are only finitely many combinatorial patterns of $C_{3}$-moves. A 'standard' pattern of a special $C_{3}$-move can be applied inside a local box on the right-hand side of the diagram of $Q_{1}$, as shown in Figure 7 .

Moreover, we can choose a quasipositive representative for this pattern, i.e. a representative whose Seifert diagram (inside the box) is quasipositive, see Figure 8. Here we remark that the two segments above and below the cross-shaped Seifert circle belong to the same Seifert circle since they are connected outside the local box.

However, this standard pattern corresponds to one specific permutation $\sigma \in S_{3}$. In order to get patterns corresponding to other permutations, we have to permute the strands inside the local box, as shown by two examples on the left-hand side of Figure 9. We observe that all of these patterns have quasipositive representatives. They are depicted on the right-hand side of Figure 9, together with their Seifert diagrams. 

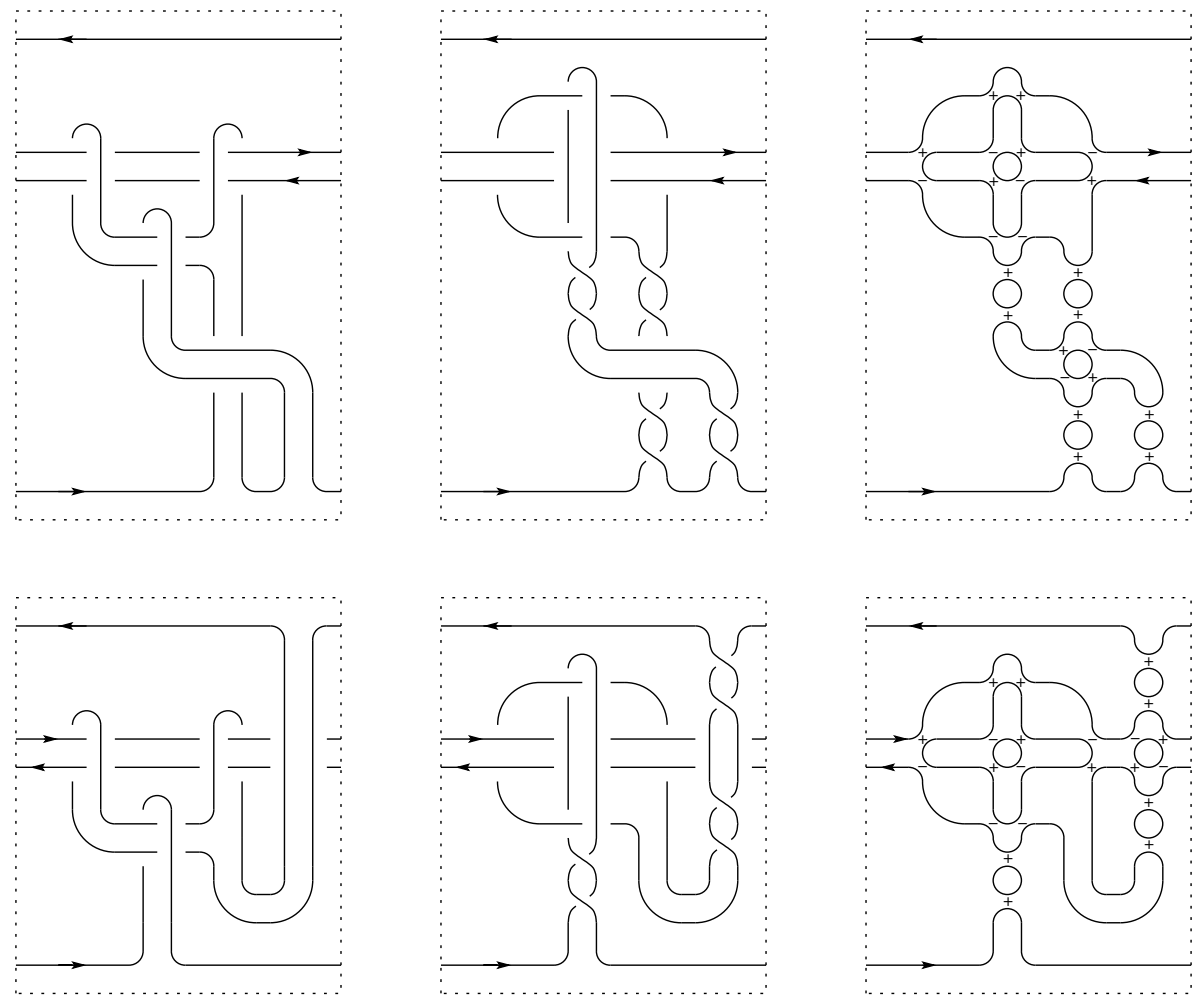

Figure 9. Permuting strands.

Since we want our $C_{3}$-move to have the same effect on $v_{3}$ as the original $C_{3}$-move that connects $Q_{1}$ and $K_{2}$, we have to arrange the product of the signs of the crossings $c_{1}, c_{2}$ and $c_{3}$. If this product does not coincide with the product of signs that comes from the original $C_{3}$-move, then we may switch it by changing four crossings between two strands inside the local box, see Figure 7, where the four crossings are encircled. This changes the sign of the crossing $c_{3}$. Thus, we can replace the knot $K_{2}$ by a quasipositive knot $\widetilde{K}_{2}$, such that

$$
v_{3}\left(\widetilde{K}_{2}\right)-v_{3}\left(Q_{1}\right)=v_{3}\left(K_{2}\right)-v_{3}\left(Q_{1}\right) .
$$

Likewise, we can replace all $C_{3}$-moves of the sequence $K_{1}=Q_{1}, K_{2}, \ldots, K_{l}=K$ by $C_{3}$-moves that take place in a clearly arranged box and preserve the quasipositivity of the knot $Q_{1}$. In this way, we obtain a sequence of quasipositive knots $\widetilde{K}_{1}=Q_{1}, \widetilde{K}_{2}, \ldots, \widetilde{K}_{l}$ and end up with a quasipositive knot $Q_{2}:=\widetilde{K}_{l}$ whose Vassiliev invariants of order two and three coincide with those of $K$.

At this point, we merely sketch how the process continues: in the $i$ th step, we arrange the Vassiliev invariants of order $i+1$ and define a quasipositive knot $Q_{i}$ whose Vassiliev invariants of order less than or equal to $i+1$ coincide with those of $K$. For this purpose, we need only observe that every combinatorial pattern of a $C_{i+1}$-move has a quasipositive representative with $i^{2}+i$ conjugating pairs of crossings, i.e. pairs of crossings satisfying the conditions (2) and (3) of quasipositive knot diagrams. The heart of such a quasipositive representative for a $C_{4}$-move is shown in Figure 10, together with its Seifert diagram. Here the 12 negative crossings can be paired with positive crossings along vertical lines. Further, we have already seen that it is easy to permute two strands inside the local box, without losing quasipositivity. At last, the product of the signs of the crossings $c_{1}, c_{2}, c_{3}, \ldots, c_{i+1}$ can always be arranged by changing the four crossings around $c_{i+1}$. After the $(n-1)$ th step, we end up with a quasipositive $\operatorname{knot} Q:=Q_{n-1}$ that has the required properties. 

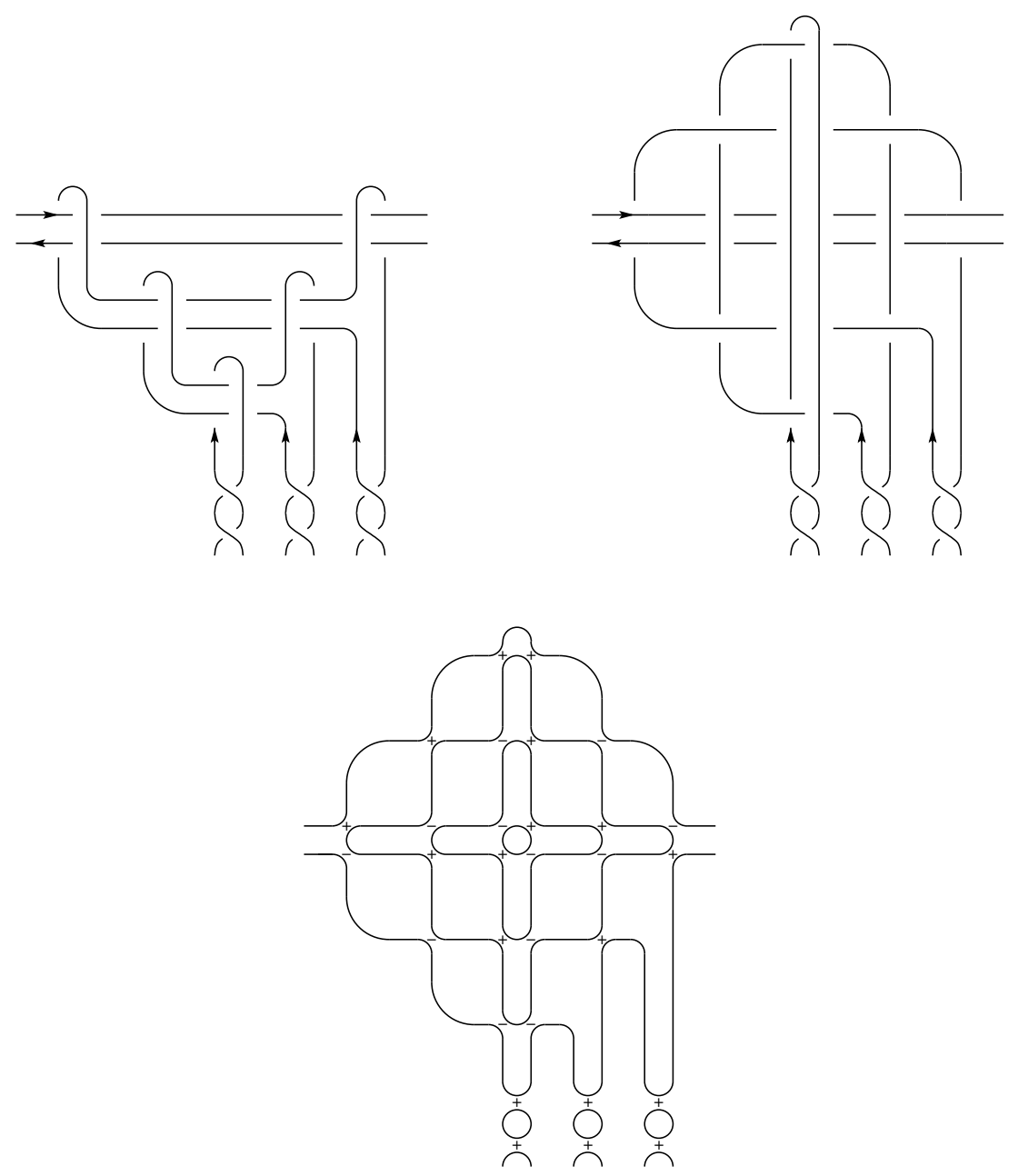

Figure 10. Quasipositive pattern for a $C_{4}$-move.

Remarks.

(i) All of the quasipositive knots $Q_{i}$ can be unknotted by a single crossing change at the clasp that appears on the left-hand side of their defining diagram (see, e.g., Figure 6). In particular, the unknotting number of $Q$ is one, unless $Q$ happens to be the trivial knot.

(ii) By Theorem 1 and Habiro's result, we conclude that every knot can be transformed into a quasipositive knot by a finite sequence of $C_{n}$-moves, for any fixed natural number $n$. It would be interesting to have a direct proof for this fact, which in turn implies Theorem 1. This would possibly simplify the construction of the desired quasipositive knots.

(iii) The knot $Q$ might even be chosen to be strongly quasipositive. However, we do not know how to prove that.

\section{ACKNowledGements}

Alexander Stoimenow pointed out an erroneous statement in the first version of this paper. I would like to thank him for that and for other useful suggestions. I am also grateful to the referees for their valuable remarks. 


\section{VASSILIEV INVARIANTS OF QUASIPOSITIVE KNOTS}

\section{REFERENCES}

Baa05 S. Baader, Slice and Gordian numbers of track knots, Osaka J. Math. 42 (2005), 257-271.

BO01 M. Boileau and S. Orevkov, Quasi-positivité d'une courbe analytique dans une boule pseudo-convexe, C. R. Acad. Sci. Paris Sér. I Math 332 (2001), 825-830.

Hab94 K. Habiro, Master thesis, University of Tokyo (1994).

Hab00 K. Habiro, Claspers and finite type invariants of links, Geom. Topol. 4 (2000), 1-83.

Ohy00 Y. Ohyama, Web diagrams and realization of Vassiliev invariants by knots, J. Knot Theory Ramifications 9 (2000), 693-701.

OTY02 Y. Ohyama, K. Taniyama and S. Yamada, Realization of Vassiliev invariants by unknotting number one knots, Tokyo J. Math. 25 (2002), 17-31.

OT99 Y. Ohyama and T. Tsukamoto, On Habiro's $C_{n}$-moves and Vassiliev invariants of order n, J. Knot Theory Ramifications 8 (1999), 15-26.

Rud82 L. Rudolph, Constructions of quasipositive knots and links. I, in Knots, braids and singularities, Plans-sur-Bex, 1982, Monographs of L'Enseignement Mathématique, vol. 31 (L'Enseignement Mathématique, Carouge, 1983).

Rud83 L. Rudolph, Algebraic functions and closed braids, Topology 22 (1983), 191-202.

Rud93 L. Rudolph, Quasipositivity as an obstruction to sliceness, Bull. Amer. Math. Soc. 29 (1993), 51-59.

Rud04 L. Rudolph, Knot theory of complex plane curves, in Handbook of Knot Theory (Elsevier, Amsterdam, 2005), 349-427.

Sto03 A. Stoimenow, Vassiliev invariants and rational knots of unknotting number one, Topology 42 (2003), 227-241.

Sebastian Baader sebastian.baader@math.ethz.ch

Department of Mathematics, ETH Zürich, Rämistrasse 101, CH-8092 Zürich, Switzerland 